

Fig. 1. Arrangement of the key elements within the subvolumes $\mathrm{III} / 7 a \cdots \mathrm{III} / 7 f$.

best available as selected by the authors; other values that may be considered reliable are frequently given in footnotes to the table. The scheme of ordering of the halide compounds in the table of crystal data is indicated in the Table of Contents, at the front of the volume.

The literature references given in the final column of the crystal data table are indicated by the last two digits of the year of the reference and the first two (occasionally three) letters of the name of the first author of the paper followed by a number $1,2,3 \ldots$ indicating the order of printing of references if there are more than one with the same year and letters. Thus, for example, $67 \mathrm{Ba} 35$ indicates a 1967 reference with the first author's name starting with the letters $\mathrm{Ba} .35$ indicates that the required reference is the thirty-fifth listed under the heading of $67 \mathrm{Ba}$ in Part $g$. In Part $g$, references are listed successively by year, and within each year alphabetically according to the two (or three) letters indicating the name of the first author of the paper. References extend through 1971 ; only six are given for 1972. The bibliography contains something like 27000 references. The final column of the crystal-data tables of Part $a$ also gives Strukturbericht - Structure Reports references where appropriate. These are indicated by the volume number (bold face) and page number; thus, 21, 360 indicates Structure Reports, Vol. 21, p. 360.

The sources of crystal data were as follows: generally up to 1961 Strukturbericht and Structure Reports, from 1962 to 1971 Chemical Abstracts, were the primary sources of literature, with the original papers being consulted wherever possible. In addition some 60 of the more important journals were searched over the period 1962 to 1971. Part $a$ contains data on 3820 substances. I made three spot checks on the accuracy of the data reproduction, the first two revealed no errors, but in the third case the reference was given incorrectly.

Although the commentary in the table of crystal data is in German, the Preface, Tables of Contents, Introduction and List of Symbols and Abbreviations are printed in English as well as German. The books are printed on good-quality paper and are generally presented with the same elegance as earlier volumes in the New Series. Notwithstanding this elegance, I am bound to observe that Crystal Data, Determinative Tables. 3rd ed. Inorganic Compounds, by J. D. H. DONNAY and H. M. ONDIK, which gives essentially the same data in a single volume, is much handier and cheaper than the eight parts of Volume 7 in this series. In many ways the duplication of labour in producing the Crystal Data and the Landolt-Börnstein series, both in collecting and checking the data and in converting it into printed books, is frightening. It seems that it is an effort that we can scarcely continue to afford in the future either scientifically or financially.

\author{
University of Waterloo \\ Waterloo \\ Ontario \\ Canada
}

W. B. PEARSON

\section{Landolt-Börnstein. Numerical data and functional relationships in science and technology. Group III. Crystal and solid state physics. Vol. 8. Epitaxy data of inorganic and organic crystals. By M. GEB- HARDT and A. Neuhaus. Pp. vii + 186. Berlin: Sprin- ger, 1972. Price: (cloth) DM 118, U.S. \$45.50.}

Volume 8 presents epitaxic data on inorganic and organic crystals and as such it extends the information provided in the crystal-data Volumes 5 to 7 . It lists the planes, directions and periods and the misfits of orientation for some 3700 epitaxic systems. The data were selected critically and cover the literature from 1836 to 1970 .

The data tables list the substrate crystal formula (and name) and often the space group or structure type, the deposited substance, the planes, directions and the period (in $\AA$ ) for both substrate and deposit, the percentage misfit of the periods based on the substrate, and the references for the data. The reference is indicated by the last two digits for the year and the first three letters of the name of the first author of the paper, followed by a number if there is more than one reference so described by year and three letters. The references are listed by year and then alphabetically by letters in a table at the end of the volume.

The arrangement of data in the epitaxic data table is indicated in the Table of Contents at the front of the volume. The main groupings of data are in four tables as follows: Inorganic Deposits on Inorganic Substrates (94 pp), Organic Deposits on Inorganic Substrates (25 pp), Inorganic Deposits on Organic Substrates (2 pp), Organic Deposits on Organic Substrates (17 pp). Each table has a number (from 2 to 5) and each epitaxic datum in the table is given a number successively, starting from 1 in each of the four tables. In the three indexes to the data at the end of the volume the substance is listed and in two columns (Substrate and Deposit) the table number ( 2 to 5 in bold face) and entry number are given according to whether the substrate occurs as substrate or deposit in the reference. In the Formula Index, inorganic substances are arranged alphabetically according to the cations. Organic compounds are listed under $\mathrm{C}$ and $\mathrm{H}$ followed by other symbols alphabetically. The other two indexes are by the names of organic compounds, and by the names of minerals.

The volume is elegantly produced and printed on good paper. The Preface, Table of Contents, Introduction and table headings are printed both in German and in English.

W. B. Pearson

University of Waterloo

Waterloo

Ontario

Canada 